Е.В. КОСТЕНКО ${ }^{1,2}$, Д.М.Н., Л.В. ПЕТРОВА ${ }^{2}$, К.М.Н.

${ }^{1}$ Российский национальный исследовательский медицинский университет им. Н.И. Пирогова, Москва

2 Московский научно-практический центр медицинской реабилитации, восстановительной и спортивной медицины

\title{
КОМПЛЕКСНАЯ РЕАБИЛИТАЦИЯ
} ПАЦИЕНТОВ С ПОСТИНСУЛЬТНОЙ
СПАСТИЧНОСТЬЮ НИЖНЕЙ КОНЕЧНОСТИ

\section{ЭФФЕКТИВНОСТЬ ПРИМЕНЕНИЯ БОТУЛОТОКСИНА (ОНАБОТУЛОТОКСИН А*)}

\begin{abstract}
Цель исследования - изучение эффективности применения препарата онаботулотоксин А (Ботокс ${ }^{\circledR}$ ) в комплексной реабилитации больных со спастичностью ноги в раннем восстановительном периоде инсульта. Материал и методы: группу наблюдения составили 31 пациент в раннем восстановительном периоде инсульта с эквиноварусной деформацией стопы с уровнем спастичности не менее 3 баллов. Все пациенты получали в составе комплексной реабилитационной программы инъекции онаботулотоксина А в общей дозе 300 ЕД. Оценивали динамику неврологического статуса, спастичности, болевого синдрома, функцию ходьбы и повседневную активность. Результаты и заключение: доказана высокая эффективность Ботокса в виде уменьшения выраженности спастичности, дискомфорта в голени и стопе, облегчения возможности самостоятельного передвижения больного, увеличения скорости ходьбы.
\end{abstract}

Ключевые слова: реабилитация, постинсультная спастичность, инсульт, эквиноварусная деформация стопы.

E.V. KOSTENKO 1, 2 , MD, L.V. PETROVA ${ }^{2}$, PhD in medicine

${ }_{1}^{1}$ Pirogov Russian National Research Medical University, Moscow, Pirogow City Clinical Hospital

2 Moscow Centre for Research and Practice in Medical Rehabilitation, Restorative and Sports Medicine of Moscow Healthcare Department COMPLEX REHABILITATION PATIENTS WITH POST-STROKE SPASTICITY OF THE LOWER LIMB: THE EFFICACY OF BOTULINUM TOXIN (ONABOTULINUMTOXIN A)

The purpose of the study was to examine the effectiveness of the drug of onabotulinumtoxin A (Botox) in complex rehabilitation of patients with post-stroke spasticity of the legs in the early rehabilitation period of stroke. Materials and methods. The study group was comprised 31 patients in the early recovery period after stroke with the presence of equino-varus deformity of the foot with the level of spasticity grade of 3 or more (MAS). All patients received as part of a comprehensive rehabilitation program injections onabotulinumtoxin a (Botox) in a total dose $300 \mathrm{ED}$. In the process of treatment was evaluated dynamics of neurological status, spasticity, pain, function of walking and everyday activity. Results and conclusion. Proven high efficacy of botulinum neurotoxin type A: reduces the severity of spasticity, the severity of the discomfort in the leg and foot; facilitated the possibility of independent movement of the patient, increases walking speed.

Keywords: rehabilitation, post-stroke spasticity, stroke, equino-varus foot deformity.

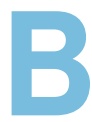

ысокая социальная значимость эффективной медицинской реабилитации пациентов с острыми нарушениями мозгового кровообращения (ОНМК) обуславливает необходимость разработки оптимальных пациент-ориентированных программ с позиций биопсихосоциальной модели инвалидизации. Наиболее частыми причинами, ограничивающими повседневную активность таких пациентов, являются парезы, когнитивные расстройства и нарушения речи. Двигательный дефицит встречается у 77-81\% пациентов после перенесенного инсульта [1]. Степень восстановления двигательных функций обратно коррелирует с исходной выраженностью неврологического дефицита (оценка по шкале NIHSS, $r=-0,44)$ и постинсультной спастичности (ПС) в раннем

" Согласно классификации Food Drug Administration (FDA). восстановительном периоде (оценка по шкале Эшворта, $r=-0,41)$ [2]. Есть данные, что спастичность, требующая специального лечения, развивается примерно у трети пациентов с инсультом. Согласно проведенным в последнее время в ряде европейских стран эпидемиологическим исследованиям выявлен разброс частоты встречаемости ПС во временном интервале от трех месяцев до года: 3 мес. - 19\%; 12 мес. - 39\%; только в руке - 15\%; только в ноге - $18 \%$; в руке и ноге - $67 \%$. По данным других авторов оценки распространенности ПС варьируют, в пределах от 4 до 42,6\%. Данные о фазах континуума ПС содержат факты ее распространенности от 4 до 27\% в течение 1-4 нед. после инсульта, от 19 до 26,7\% в течение 1 - 3 мес. и от 17 до 42,6\% > 3 мес. после перенесенного инсульта [1]. По данным разных исследований, распространенность спастичности через 12 мес. после инсульта составляет 36,049,7\% [3]. Наиболее значимыми для восстановления дви- 
гательных функций являются первые 6-12 мес. после инсульта [4]. ПС имеет тенденцию к нарастанию в течение первых месяцев после инсульта, что ведет к формированию контрактур, затрудняет самообслуживание, делает уход за пациентом обременительным и оказывает отрицательное влияние на качество жизни больных и их семей [5-8]. Наличие спастичности требует проведения дополнительных реабилитационных мероприятий, что приводит к увеличению прямых затрат, связанных с ОНМК. Так, прямые затраты на лечение лиц с ПС, выживших в течение 12 мес. после инсульта, в 4 раза превышают таковые при терапии пациентов без спастичности [9].

Цель реабилитационных мероприятий индивидуальна в каждом конкретном случае и достигается посредством реализации принципа комплексного пациент-ориентированного подхода для улучшения пассивной и по возможности активной функций; предотвращения формирования контрактур; уменьшения боли и мышечного спазма; улучшения походки, повседневной активности, гигиены и ухода; улучшения качества жизни пациента.

Современные антиспастические лекарственные средства не обладают селективным механизмом действия, и их применение может приводить к ухудшению общего функционального состояния пациента. У некоторых больных эти препараты уменьшают силу здоровых мышц, не оказывая эффекта на ПС [10-14]. Альтернативной стратегией лечения спастичности является локальный химический нейролизис алкоголем или фенолом, что может вызывать потерю чувствительности и дизестезию, затрудняющие восстановление физиологического паттерна ходьбы [15-17]. При лечении ПС с доказанной эффективностью применяется локальное введение БТА, имеющее ряд несомненных преимуществ [18-25]: лечение хорошо переносится и не связано с риском серьезных осложнений; возможность индивидуального выбора таргетных мышц для инъекций и дозы препарата. Ботулинотерапия представляет собой необходимую интегрированную часть комплексного реабилитационного лечения пациентов с ПС [6, 26-28].

В настоящее время известны следующие клиникодиагностические типы ПС ноги: эквиноварусная стопа (подошвенное сгибание и пронация стопы); согнутое бедро- проксимальный флексорный спазм; приведенное бедро; клешневидные пальцы; гиперактивность большого пальца; экстензорная спастичность; паттерн жесткого колена; согнутое колено.

Хотя пациенты испытывает дискомфорт при ходьбе в связи с разогнутым коленом, такая позиция позволяет фиксировать колено и выравнивать баланс при ходьбе, обеспечивая функцию опоры. Функциональные возможности нижней конечности у пациента с постинсультным парезом хуже при наличии выраженной спастичности, чем при ее легкой степени. Подошвенная флексия и ротация стопы у пациентов со спастичной нижней конечностью вызывают нарушение опороспособности, подволакивание стопы и подгибание пальцев при ходьбе, снижение скорости движения, формирование болевого синдрома и контрактур [7, 18]. Основная цель лечения ПС ноги состоит в улучшении функциональных возможностей, самообслуживания, уменьшении боли и дискомфорта, облегчении ухода и устранении имеющегося косметического дефекта [8, 21, 23].

В литературе представлено значительное количество рандомизированных двойных слепых плацебо-контролируемых исследований, показавших эффективность препаратов БТА при лечении ПС верхней конечности [27-35, 36-38]. Работ, посвященных лечению ПС нижней конечности, не так много. В основном наблюдения в этой области, опубликованные на протяжении последних десятилетий, базируются на небольших выборках пациентов. Большинство подобных исследований ставило целью выяснить, насколько уменьшается выраженность эквиноварусной установки стопы при введении БТА и улучшается функциональная активность конечности $[15,16,23,32$, 39-44].

Анализ результатов 9 двойных слепых плацебо-контролируемых исследований показал высокий профиль безопасности БТА [14]. Продемонстрировано существенное уменьшение спастичности после однократного введения Ботокса (300 ЕД) и спастичности в голеностопном суставе по сравнению с группой плацебо-терапии ( 0,001) [38]. Данные рандомизированных контролируемых исследований безопасности и эффективности ботулинотерапии при лечении пациентов со спастичностью ноги показали, что эффект сохраняется дольше при использовании больших доз препарата (табл. 1). Длительное существование спастичности ассоциировано с худшими функциональными возможностями конечности и неудовлетворительным восстановлением.

Целью исследования было оценить эффективность препарата Ботокс ${ }^{\circledR}$ в комплексной реабилитации больных с постинсультной спастичностью ноги в раннем восстановительном периоде после инсульта.

\section{МАТЕРИАЛ И МЕТОДЫ}

Под наблюдением находились 31 пациент в раннем восстановительном периоде инсульта, 17 женщин и 14 мужчин, в возрасте от 41 до 75 лет (средний - 58,4 \pm 6,4 года). Давность перенесенного инсульта колебалась от 28 до 182 дней, составляя в среднем 98,59 \pm 31,9 дня; преобладали пациенты с давностью инсульта 60-120 дней (71,0\%). Ишемический инсульт был у 71,0\% больных, геморрагический - у 29,0\%. У всех пациентов наблюдалась полушарная локализация очага поражения: в правом полушарии - у 45,2\%; в левом - у 54,8\%. Основными причинами развития инсульта были артериальная гипертензия, атеросклероз и их сочетание.

Критериями включения в исследование являлись: возраст от 20 до 80 лет; масса тела не менее 50 кг; наличие эквиноварусной деформации стопы (плантарная флексия стопы) с уровнем спастичности 3 балла и более по модифицированной шкале Эшворта (MAS); ранний восстановительный период инсульта. Критерии исключения: наличие мышечных контрактур, предшествующее лечение БТА, блокадами фенолом или алкоголем, или другими 


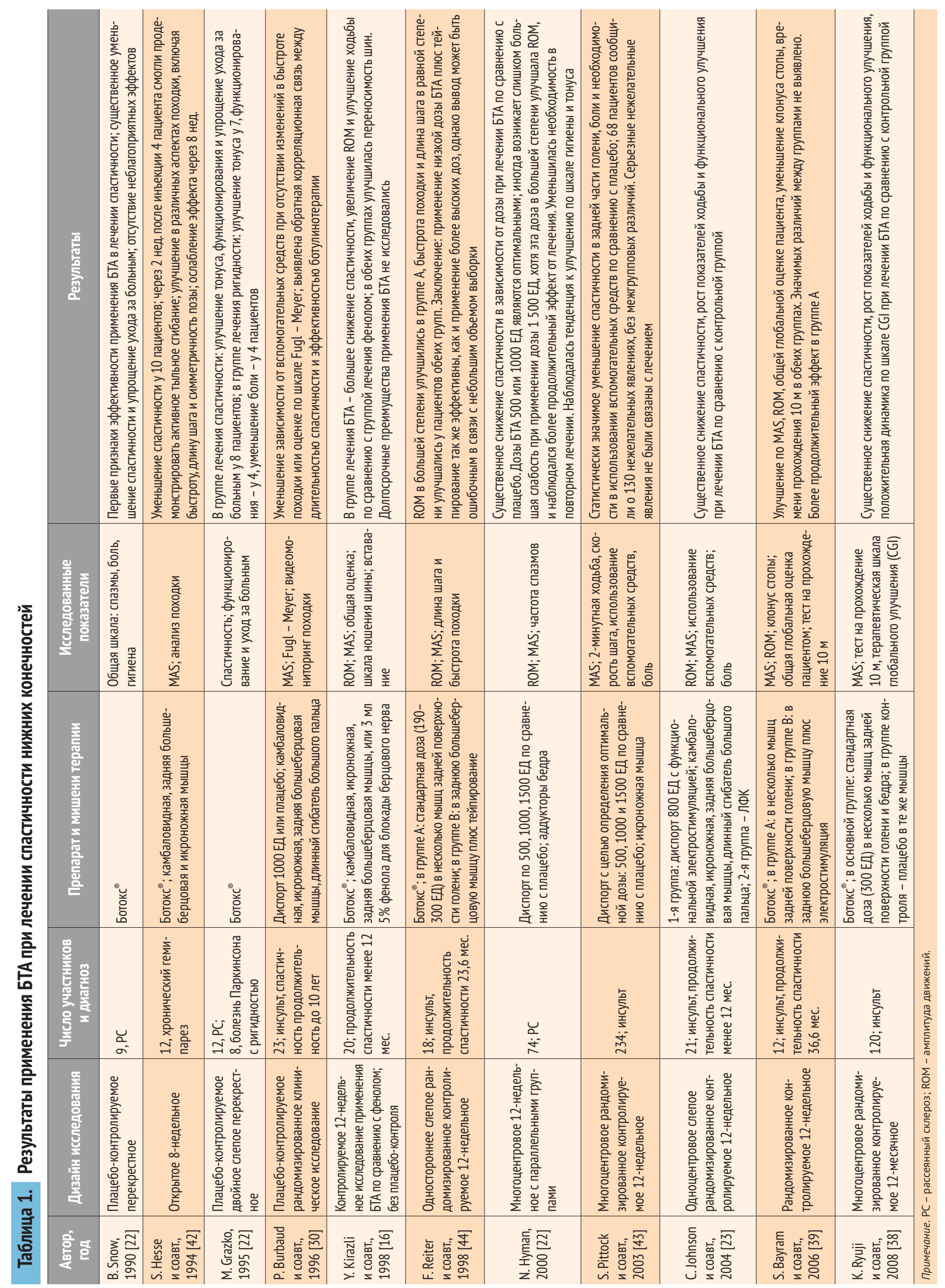


инъекциями в двигательные точки по поводу спастичности нижней конечности; нижний парапарез; глубокая атрофия целевых для инъекций мышц; наличие баклофеновой помпы; беременность и лактация.

Все пациенты дали информированное согласие на участие в исследовании.

По типу спастических нарушений все больные были разделены на 3 группы: 1-я группа - 19 (61,3\%) пациентов с изолированной эквиноварусной установкой стопы; 2-я группа - 7 (22,6\%) пациентов с эквиноварусной установкой стопы в сочетании с подошвенным сгибанием пальцев стопы, большого пальца стопы; 3-я группа - 5 $(16,1 \%)$ пациентов с эквиноварусной установкой стопы в сочетании с приведением бедра кнутри (повышение тонуса медиальной группы мышц).

Всем пациентам помимо общеклинического обследования проводилась оценка неврологического статуса с использованием следующих инструментов: 1) шкала Эшворта для оценки спастичности (от 0 до 4 баллов); 2) визуально-аналоговая шкала (ВАШ) оценки боли, связанной со спастичностью в ноге; 3) шкала общей оценки исследователем и пациентом затруднений при ходьбе пациентом и исследователем в баллах от 0 (совсем не трудно) до 5 (крайне трудно) оценивается возможность передвижения больного с и без использования вспомогательного средства на протяжении последних 7 дней перед визитом; 4) тест на прохождение 10 м - исследуются скорость прохождения 10 м по ровной поверхности в привычном темпе и максимально возможная скорость передвижения, при этом допустимы опора на ассистента и/или использование вспомогательных средств; 5) глобальная оценка трудностей при ходьбе - от 1 (отсутствие признака) до 4 (самые серьезные нарушения) баллов, оцениваются характеристики ходьбы: неустойчивость, необходимость прилагать усилия при ходьбе, впечатление наличия боли, затруднения при передвижении в целом; 6) шкала общего клинического впечатления (CGI) - респондерами считались те больные, физическое состояние которых на определенный момент терапии соответствовало 1 или 2 баллам; 7) шкала достижения пациентом основных целей (GAS), использовалась однократно в конце исследования. Оценка по этой шкале выставляется следующим образом: если пациент достигает ожидаемого результата - ставят 0; если достигается результат лучше ожидаемого - ставят +1 (немного лучше) или +2 (значительно лучше); если достигается результат хуже ожидаемого - ставят -1 (немного хуже) или -2 (значительно хуже).

В структуру комплекса реабилитационных мероприятий входило: медикаментозное лечение, физиотерапия (включая методы электростимуляции), лечебная физкультура (включая позиционирование и растяжение), механотерапия (включая использование систем с биологической обратной связью (БОС-систем) и роботизированных техник), рефлексотерапия, локальные инъекции ботулинического нейротоксина типа А (БТА).

Всем больным однократно проводились инъекции БТА $\left(\right.$ Ботокс ${ }^{\circledR}$ ) по стандартной схеме в общей дозе 300 ЕД.
Общая суммарная доза БТА 300 ЕД распределялась по стандартной схеме в каждой из 3 групп пациентов. Инъекции проводили в 3 точки в области двигательных зон мышц по анатомическим ориентирам под контролем электромиографии (табл. 2).

\section{Таблица 2. Дозировки введения БТА (Ботокс ${ }^{\circledR}$ ) у обследованных пациентов}

\begin{tabular}{l|c|l}
\hline $\begin{array}{c}\text { Иньецируемая } \\
\text { мышща }\end{array}$ & $\begin{array}{c}\text { Дозировка } \\
\text { Ботокса, } \\
\text { ЕД }\end{array}$ & \multicolumn{1}{|c}{$\begin{array}{c}\text { Анатомические ориентиры } \\
\text { точек введения, } \\
\text { количеспво точек иньекций }\end{array}$} \\
\hline $\begin{array}{l}\text { мastrocnemius } \\
\text { медиальная головка }\end{array}$ & 75 & $\begin{array}{l}\text { Поверхностная мышца на медиаль- } \\
\text { ной поверхности задней части голе- } \\
\text { ни, 3 точки }\end{array}$ \\
\hline $\begin{array}{l}\text { Gastrocnemius } \\
\text { латеральная головка }\end{array}$ & 75 & $\begin{array}{l}\text { Поверхностная мышца на латераль- } \\
\text { ной поверхности задней части голени, } \\
\text { 3 точки }\end{array}$ \\
\hline Soleus & 75 & $\begin{array}{l}\text { Задняя поверхность голени, посере- } \\
\text { дине и внизу между мышечными } \\
\text { брюшками икроножной мышцы, } \\
\text { 3 точки }\end{array}$ \\
\hline Tibialis posterior & 75 & $\begin{array}{l}\text { Посередине задней части голени, глу- } \\
\text { боко за малоберцовой костью и в } \\
\text { углублении между большеберцовой } \\
\text { и малоберцовой костями, 3 точки }\end{array}$ \\
\hline
\end{tabular}

Изменения биомеханики походки у пациентов показали неспецифическую симптоматику, являющуюся симптомокомплексом снижения темпа шага. Из специфичных характеристик были обнаружены: функциональные последствия недостаточности четырехглавой мышцы бедра, которая проявляется в виде пассивного замыкания коленного сустава и недостаточности трехглавой мышцы голени, выражающейся в снижении амплитуды отталкивания продольной составляющей реакции опоры и уменьшении амплитуды подошвенного сгибания голеностопного сустава; асимметрия периодов цикла шага; снижение стабильности, что сопровождается отклонением центра давления (ЦД) на здоровую сторону; положение ЦД в сагиттальной плоскости характеризовалось смещением вперед на величину около 9 мм; колебания ЦД превышали норму как во фронтальной, так и в сагиттальной плоскости; площадь статокинезиограммы превышала норму.

Оценку неврологического статуса, спастичности и двигательных функций ноги проводили до начала терапии (1-й визит) и через 2, 4, 8, 12 и 16 нед. после лечения (2-5-й визиты). Безопасность Ботокса оценивали в соответствии с сообщениями о побочных эффектах. Опрос по поводу наличия побочных эффектов и осмотр для выявления системных нежелательных явлений осуществляли при каждом визите больного. Лабораторные тесты проводили на момент скрининга (1-й визит), через 4 и 16 нед. в процессе наблюдения пациентов.

Результаты исследований подвергались статистической обработке с использованием стандартных функций 
программ Microsoft Excel и Statistica 6.0. Достоверность различий определяли с помощью t-теста Стьюдента и методов непараметрической статистики (критерий $\mathrm{X}^{2}$ ). Для выявления взаимосвязи двух признаков применялся непараметрический корреляционный анализ по Спирмену. Различия считались достоверными при $p<0,05$.

\section{РЕЗУЛЬТАТЬ}

Все пациенты полностью закончили исследование. Через 2 нед. после инъекции было выявлено уменьшение показателей спастичности по шкале Эшворта, однако эти изменения не достигали степени статистической значимости. Через 4 нед. после начала лечения наблюдалось достоверное снижение степени спастичности в дистальном отделе нижней конечности. Максимальный клинический эффект отмечался в период 4-8 нед. после инъекции ( $p<0,05$, табл. 3), через 16 нед. после начала лечения степень спастичности нарастала, не достигая исходной $(p>0,05)$.

Таблица 3. Динамика показателя спастичности по шкале Эшворта (баллы) под влиянием лечения через 2, $4,8,12$ и 16 нед. (M \pm m)

Срок после иньекции БТА $\quad$ Спастичность в голеностопном суставе

\begin{tabular}{c|c}
\hline До лечения & $3,8 \pm 0,22$ \\
\hline Через 2 нед. & $3,63 \pm 0,47$ \\
\hline Через 4 нед. & $2,75 \pm 0,38^{*}$ \\
\hline Через 8 нед. & $2,5 \pm 0,5^{*}$ \\
\hline Через 12 нед. & $3,38 \pm 0,47$ \\
\hline Через 16 нед. & $3,68 \pm 0,47$ \\
\hline
\end{tabular}

Примечание. Здесь и в таблицах 4 и 5: * - отличия достоверны по сравнению с исходным уровнем $(\mathrm{p}<0,05)$.

При оценке боли, связанной со спастичностью в ноге, до лечения 58\% пациентов оценивали боль в голеностопе и стопе как терпимую; 22,5\% - как боль умеренной интенсивности и 16,1\% - как нестерпимую; в 3,4\% случаев жалоб на боль или дискомфорт в нижней конечности не предъявлялось. Средний показатель болевого синдрома составил 6,18 $\pm 0,41$ балла. Во всех случаях боль уменьшалась ко 2-й нед. после проведения инъекции, и по окончании курса терапии все больные отмечали значительное субъективное улучшение. Статистически значимое снижение интенсивности болевого синдрома по ВАШ наблюдалось через 4 нед. после инъекции $(2,9 \pm 1,1$ балла, $p<0,05)$. Интенсивность боли к моменту окончания исследования уменьшилась до 3,75 \pm 0,78 балла по сравнению с исходной оценкой в 6,18 \pm 0,41 балла. У всех пациентов с 4-й по 8-ю нед. после введения Ботокса наблюдалось статистически значимое урежение частоты возникновения болевого синдрома (табл. 4).
Таблица 4. Динамика выраженности боли, связанной со спастичностью в ноге, и затруднений при ходьбе под влиянием лечения через $2,4,8,12$ и 16 нед. после начала терапии у обследованных больных (баллы, $\mathrm{M} \pm \mathrm{m}$ )

\begin{tabular}{c|c|c|c}
\hline $\begin{array}{c}\text { Срок после } \\
\text { иньекии БТА }\end{array}$ & $\begin{array}{c}\text { Оценка } \\
\text { по ВАІІ }\end{array}$ & $\begin{array}{c}\text { Частота болевого } \\
\text { синдрома }\end{array}$ & $\begin{array}{c}\text { Запруднения } \\
\text { при ходьбе }\end{array}$ \\
\hline До лечения & $6,18 \pm 0,41$ & $3,9 \pm 0,22$ & $4,13 \pm 0,15$ \\
\hline Через 2 нед. & $3,5 \pm 0,7$ & $3,63 \pm 0,47$ & $3,86 \pm 0,17$ \\
\hline Через 4 нед. & $2,92 \pm 0,9^{*}$ & $2,75 \pm 0,38^{*}$ & $3,35 \pm 0,2^{*}$ \\
\hline Через 8 нед. & $2,95 \pm 0,85$ & $2,5 \pm 0,5^{*}$ & $3,17 \pm 0,21^{*}$ \\
\hline Через 12 нед. & $3,63 \pm 0,82$ & $3,38 \pm 0,47$ & $3,78 \pm 0,44$ \\
\hline Через 16 нед. & $3,75 \pm 0,78$ & $3,63 \pm 0,47$ & $3,78 \pm 0,44$ \\
\hline
\end{tabular}

Аналогичная динамика наблюдалась при оценке пациентом и исследователем затруднений при ходьбе. Если исходно большая часть пациентов отмечали существенные трудности при передвижении, в т. ч. с использованием вспомогательных средств, то к 4-й нед. терапии наблюдалось статистически значимое улучшение качества ходьбы (небольшие или умеренные трудности). Подобный клинический эффект сохранялся до 8-й нед. терапии с последующим возвращением показателей к исходным параметрам (табл. 4). Уменьшение выраженности затруднений при ходьбе было, вероятно, обусловлено снижением как спастичности в стопе, так и степени дискомфорта/боли.

До лечения при прохождении пациентом 10 м по ровной поверхности отмечалось существенное снижение скоростных показателей ходьбы. К 4-й нед. терапии наблюдалось статистически значимое нарастание скорости ходьбы, в дальнейшем при сохранении положительной динамики достоверных различий этого показателя по сравнению с исходным уровнем получено не было (табл. 5).

Изучение глобальной оценки трудностей при ходьбе по четырем основным показателям выявило, что через 4

\section{Таблица 5. Динамика показателей скорости ходьбы при проведении теста на прохождение 10 м под влия- нием лечения через 2, 4, 8, 12 и 16 нед. после начала терапии у обследованных больных (M $\pm m, c)$}

\begin{tabular}{c|c|c}
\hline $\begin{array}{c}\text { Срок после } \\
\text { иньекции БТА }\end{array}$ & $\begin{array}{c}\text { Скорость ходьбы } \\
\text { в привычном темпе }\end{array}$ & $\begin{array}{c}\text { Максимально возможная } \\
\text { скорость ходьбы }\end{array}$ \\
\hline До лечения & $73,6 \pm 9,2$ & $67,5 \pm 12,3$ \\
\hline Через 2 нед. & $71,8 \pm 10,1$ & $66,6 \pm 9,6$ \\
\hline Через 4 нед. & $58,2 \pm 12,4^{*}$ & $53,6 \pm 11,6$ \\
\hline Через 8 нед. & $64,5 \pm 9,85$ & $58,8 \pm 11,5$ \\
\hline Через 12 нед. & $63,63 \pm 13,82$ & $60,3 \pm 7,4$ \\
\hline Через 16 нед. & $69,75 \pm 12,7$ & $62,6 \pm 10,7$ \\
\hline
\end{tabular}


нед. после начала лечения по всем этим показателям наблюдалось статистически значимое улучшение. Эффект был максимально выражен через 4-12 нед. после введения препарата и уменьшался к концу 4-го мес. после инъекции БТА. При тестировании через 16 нед. показатели трудностей, связанных с ходьбой, ухудшились, но не вернулись к исходным значениям (различия не достоверны) (табл. 6).

K моменту завершения исследования терапевтический эффект разной степени выраженности при балльной оценке по шкале CGI наблюдали у 28 (90,3\%) пациентов, В т. ч. очень хороший эффект - у 3 (9,6\%), хороший - у 15 $(48,4 \%)$ и удовлетворительный - у 10 (32,5\%). Для выяснения механизма формирования лечебного эффекта при применении различных методов восстановительного лечения у больных с высокой степенью ПС ноги был проведен корреляционный анализ между изучаемыми показателями через 4 и 16 нед., который выявил связь между показателями состояния мышечного тонуса через 4 нед. после инъекции БТА и давностью заболевания ( $r=0,58 ; p$ < 0,01). Применение у больных комплекса реабилитационных мероприятий, включая ботулинотерапию, способствует перераспределению ЦД на паретичную ногу и восстановлению двигательного стереотипа, что объективно подтверждается данными компьютерной стабилометрии: уменьшением смещения ЦД во фронтальной плоскости, площади и длины пути стабилограммы.
К моменту окончания исследования суммарная оценка по шкале GAS для первичной цели терапии статистически значимо увеличилась по сравнению с исходными показателями с 27,2 (22,8-32,5) до 36,4 (29,6-40,6) балла, уровень изменения внутри группы - 9,6 (0-12,3) балла. У большинства пациентов, по их мнению, имелось функциональное улучшение состояния нижней конечности ( $p=0,011)$, однако данная динамика была не такой выраженной при оценке по шкале Эшфорта ( $p=$ $0,8)$ и с помощью теста на прохождение 10 м ( $p=0,77)$. Таким образом, в исследовании продемонстрирована высокая значимость оценки по шкале достижения цели, которая может быть более чувствительной в сравнении с другими тестами.

Серьезных нежелательных явлений, угрожающих жизни, или инвалидизирующих побочных эффектов, связанных с лечением Ботоксом, отмечено не было. Общая слабость и системные побочные эффекты не наблюдались. Нежелательные явления легкой или средней степени выраженности, вероятно связанные с использованием препарата, были зарегистрированы у 5 $(16,1 \%)$ больных и представлены слабостью в инъецируемой конечности легкой и умеренной степени - у 3 (9,7\%), миалгией - у 1 (3,2\%), локальной болезненностью в месте инъекции - также у 1 (3,2\%) пациента. Через 4 нед. наблюдения данные побочные эффекты не регистрировались.

\section{БоTOKC}

Направленное Сенсомоторное Действие

\section{Время бесценно. Не упусти моменто}

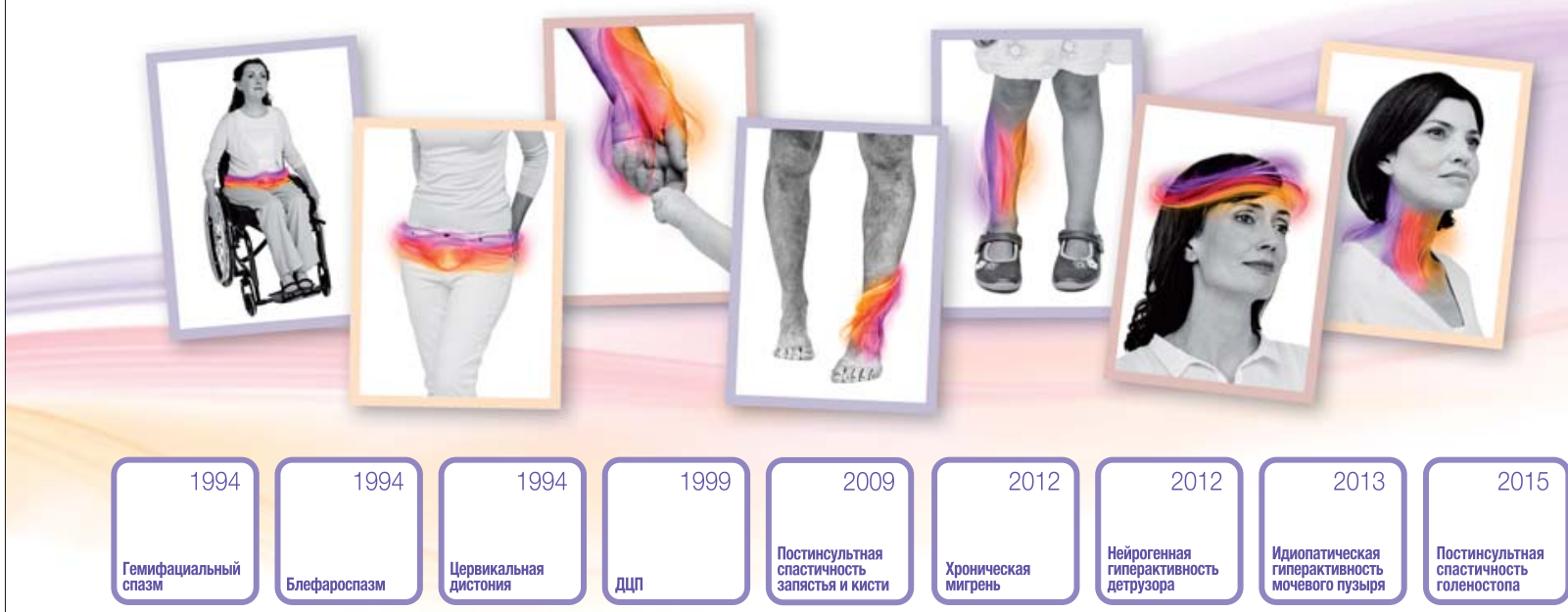

Ботокс - П N 011936/01, Аллерган Фармасьютикэлз Айэрлэнд

Перед назначением препарата, пожалуйста, ознакомьтесь с полной инструкцией по медицинскому применению.

Получить дополнительную информацию, сообщить о нежелательной реакции при применении, а также направить претензию ккачес

по адресу: ООО «Аллерган СНГ С.А.Р.Л.» Российская Федерация, 115191, г. Москва, Холодильный переулок, д. 3, корп. 1, стр. 4.

по телефону: 8-800-250-98-25 (звонок по России бесплатный) по факсу: 8-800-250-98-26 по әлектронной почте: MW-Medlnfo@Allergan.com

1. Даты опобрения показаний кприменению препарата Ботокс в в Российской Федерации 
Таблица 6. Динамика показателей шкалы глобальной оценки трудности при передвижении на фоне терапии БТА (\% пациентов)

\begin{tabular}{|c|c|c|c|c|}
\hline $\begin{array}{c}\text { Срок после } \\
\text { иньекции БТА }\end{array}$ & $\begin{array}{l}\text { Нестабильность, } \\
\text { шаткость походки }\end{array}$ & $\begin{array}{c}\text { Приложение } \\
\text { усилий }\end{array}$ & Боль & $\begin{array}{c}\text { Общие } \\
\text { трудности } \\
\text { при ходьбе }\end{array}$ \\
\hline $\begin{array}{l}\text { До лечения } \\
4 \text { балла } \\
3 \text { балла } \\
2 \text { балла } \\
1 \text { балл }\end{array}$ & $\begin{array}{c}- \\
51,6 \\
48,4 \\
-\end{array}$ & $\begin{array}{c}- \\
80,6 \\
19,4 \\
-\end{array}$ & $\begin{array}{c}6,5 \\
25,8 \\
67,7 \\
-\end{array}$ & $\begin{array}{c}- \\
48,4 \\
51,6 \\
-\end{array}$ \\
\hline $\begin{array}{l}\text { Через } 2 \text { нед. } \\
4 \text { балла } \\
3 \text { балла } \\
2 \text { балла } \\
1 \text { балл } \\
p \\
x^{2}\end{array}$ & $\begin{array}{c}- \\
45,2 \\
54,8 \\
- \\
0,19 \\
1,67\end{array}$ & $\begin{array}{c}- \\
67,7 \\
32,3 \\
- \\
1,0 \\
0,0\end{array}$ & $\begin{array}{c}- \\
32,3 \\
67,7 \\
- \\
0,46 \\
0,53\end{array}$ & $\begin{array}{c}- \\
42,0 \\
58,0 \\
- \\
0,07 \\
3,13\end{array}$ \\
\hline $\begin{array}{l}\text { Через } 4 \text { нед. } \\
4 \text { балла } \\
3 \text { балла } \\
2 \text { балла } \\
1 \text { балл } \\
p \\
X^{2} \\
\end{array}$ & $\begin{array}{c}- \\
32,3 \\
67,7 \\
- \\
0,046 \\
3,96\end{array}$ & $\begin{array}{c}- \\
52,0 \\
48,0 \\
- \\
0,03 \\
4,33\end{array}$ & $\begin{array}{c}- \\
9,7 \\
83,8 \\
6,5 \\
0,03 \\
4,33\end{array}$ & $\begin{array}{c}- \\
25,8 \\
74,2 \\
- \\
<0,001 \\
7,03\end{array}$ \\
\hline $\begin{array}{l}\text { Через } 8 \text { нед. } \\
4 \text { балла } \\
3 \text { балла } \\
2 \text { балла } \\
1 \text { балл } \\
p \\
x^{2}\end{array}$ & $\begin{array}{c}- \\
32,3 \\
67,7 \\
- \\
0,046 \\
3,96\end{array}$ & $\begin{array}{c}- \\
58,0 \\
42,0 \\
- \\
0,46 \\
0,53\end{array}$ & $\begin{array}{c}- \\
9,7 \\
83,8 \\
6,5 \\
0,03 \\
4,33\end{array}$ & $\begin{array}{c}- \\
25,8 \\
74,2 \\
- \\
0,028 \\
4,8\end{array}$ \\
\hline $\begin{array}{l}\text { Через } 12 \text { нед. } \\
4 \text { балла } \\
3 \text { балла } \\
2 \text { балла } \\
1 \text { балл } \\
p \\
X^{2}\end{array}$ & $\begin{array}{c}- \\
45,2 \\
54,8 \\
- \\
0,19 \\
1,67\end{array}$ & $\begin{array}{c}- \\
58,0 \\
42,0 \\
- \\
0,46 \\
0,53\end{array}$ & $\begin{array}{c}- \\
9,7 \\
83,8 \\
6,5 \\
0,03 \\
4,33\end{array}$ & $\begin{array}{c}- \\
35,5 \\
64,5 \\
- \\
0,058 \\
2,77\end{array}$ \\
\hline $\begin{array}{l}\text { Через } 16 \text { нед. } \\
4 \text { балла } \\
3 \text { балла } \\
2 \text { балла } \\
1 \text { балл } \\
p \\
x^{2}\end{array}$ & $\begin{array}{c}- \\
48,4 \\
51,6 \\
- \\
0,75 \\
0,1\end{array}$ & $\begin{array}{c}- \\
67,7 \\
32,3 \\
- \\
1,0 \\
0,0\end{array}$ & $\begin{array}{c}3,2 \\
25,8 \\
67,7 \\
3,2 \\
1,0 \\
0,0\end{array}$ & $\begin{array}{c}- \\
45,1 \\
54,9 \\
- \\
0,5 \\
0,44\end{array}$ \\
\hline
\end{tabular}

\section{ОБСУЖДЕНИЕ}

Результаты проведенного исследования согласуются с данными предыдущих открытых испытаний Ботокса в терапии ПС нижней конечности $[15,16,23,32,39$, 40 -44]. Показано, что Ботокс ${ }^{\circledR}$, применяемый в составе комплексной терапии, статистически значимо уменьшает мышечный тонус у больных с выраженной ПС ноги. Лечение препаратом Ботокс ${ }^{\circledR}$ в дозе 300 ЕД было эффективным и безопасным, значимой слабости конечности и системных побочных эффектов не наблюдалось. Клинический эффект прослеживался через 2 нед. после инъекции БТА, достигая максимума к 4-й нед, и затем постепенно угасал к 16-й нед. наблюдения. Результаты использования рейтинговых шкал не всегда были сопоставимы. Так, статистически значимое улучшение по шкале спастичности Эшворта наблюдалось к 4-й и 8-й нед. после введение препарата с последующим ухудшением показателей тестирования. Результаты теста на прохождение 10 м оказались статистически значимыми только на 4-й нед. после проведения ботулинотерапии.

Спастичность часто ассоциируется с мышечной болью [46]. Обследованные пациенты с выраженной спастичностью предъявляли жалобы на боль или дискомфорт в паретичной конечности. После инъекции Ботокса, наряду со снижением мышечного тонуса с 4-й по 12-ю нед. наблюдалось статистически значимое снижение боли и дискомфорта в ноге. Таким образом, по результатам проведенного исследования можно сделать вывод о высокой эффективности Ботокса в лечении боли или болезненных мышечных спазмов, связанных со спастичностью, что согласуется с данными других авторов.

В заключение следует отметить, что одной из современных тенденций в терапии инсульта является следование концепции ранней комплексной реабилитации. Для изменения паттерна спастичности и стереотипа ходьбы требуется проведение длительного восстановительного лечения (в нашем случае непрерывная реабилитация длилась 16 нед) с привлечением специалистов мультидисциплинарной бригады: неврологов, психотерапевтов, физио-, рефлексотерапевтов, инструкторов лечебной физкультуры. Профилактика и снижение спастичности позволяют улучшить функциональную активность ноги, предупредить формирование контрактур и аномальных положений нижней конечности, снижающих качество жизни больных, перенесших инсульт. Проведенный корреляционный анализ подтвердил, что с увеличением давности заболевания степень выраженности спастичности нарастает, что затрудняет и снижает эффективность реабилитации пациента, перенесшего инсульт.

В соответствии с полученными в исследовании результатами можно сделать следующие выводы:

- Применение Ботокса в комплексной реабилитации больных с ПС нижней конечности в раннем восстановительном периоде инсульта достоверно снижает фокальную спастичность голеностопа через 4 нед. от начала лечения.

- Введение Ботокса позволяет не только снизить тонус мышц нижней конечности у больных с выраженной спастичностью, но и улучшить двигательную функцию ноги, качество ходьбы, снизить степень выраженности болевого синдрома в пораженной конечности.

- Минимальный терапевтический эффект обнаруживается уже через 2 нед. лечения, и по мере продолжения терапии наблюдается его нарастание с максимумом к 4-8й нед.

— К 16-й нед. после проведенной инъекции отмечаются истощение эффекта и нарастание мышечного тонуса.

- Ботокс ${ }^{\circledR}$ имеет хороший профиль безопасности. 


\section{ЛИТЕРАТУРА}

1. Wissel J, Manack A, Brainin M. Toward an epidemiology of poststroke spasticity. Neurology, 2013, 3(2): 13-19.

2. Феррахов А.З., Хабиров Ф.А., Ибрагимов М.Ф., Хайбуллин Т.И., Гранатов Е.В. Комплексная реабилитация больных, перенесших инсульт, на этапах «стационар - реабилитационный центр - поликлиника». Неврологический вестник, 2012, 44(4): 3-8. / Ferrakhov A.Z., Khabirov F.A., Ibragimov M.F., Khaibullin T.I. Granatov E.V. Complex rehabilitation of patients who suffered from a stroke, at the stages "inpatient department - rehabilitation center polyclinic". Nevrologicheskiy Vestnik, 2012, 44 (4): 3-8.

3. Ward AB, Aguilar M, De Beyl Z, Gedin S, Kanovsky P, Molteni F, et al. Use of botulinumtoxin type $A$ in management of adult spasticity-a European consensus statement. J Rehabil Med, 2003, 35: 98-99.

4. Парфенов В.А. Постинсультная спастичность и ее лечение. РМЖ, 2006, 1, 9(261): 689-693.

5. Urban P, Wolf T, Uebele M. Occurence and clinical predictors of spasticity after ischemic stroke. Stroke, 2010, 41(9): 2016-2020.

6. Picelli A, Tamburin S, Gajofatto F, Zanette G, Praitano M, Saltuari L et al. Association between Severe Upper Limb Spasticity and Brain Lesion Location in Stroke Patients. Biomed Research International, 2014, 1-6.

7. Reichel G. Therapielietfa den Spastik. 3. Auflage-Bremen, UNI-MED, 2013: 190.

8. Watkins C, Leathley M, Gregson J, Moore A, Smith T, Sharma A. Prevalence of spasticity post stroke. Clinical Rehabil, 2002, 16: 515-552.

9. Lundström E, Smits A, Borg J, Terént A. Comment in Stroke, 2010, 41(7): e470, author reply e471. Stroke, 2010, 41(2): 201-202.

10. Батышева Т.Т., Бойко А.Н., Костенко Е.В. Методологические основы лечения спастичности. Медико-социальные аспекты лечения спастичности в практике врачаневролога. Материалы научно-практической конференции. М., 2010: 6-9. / Batysheva Т.T. Boyko A.N., Kostenko E.V. Methodological fundamentals of spacticity therapy. Medical and social aspects of spasticity therapy in the prac tice of a neurologist. Materials of scientific and practical conference. M., 2010; 6-9.

11. Гусев Е.И., Гехт А.Б., Гаптов В.Б., Тихопой Е.В. Реабилитация в неврологии. М., 2000: 52./ Gusev E.I., Gekht A.B., Gaptov V.B., Tikhopoy E.V. Rehabilitation in neurology. M., 2000: 52.

12. Воробьева О.В. Нарушение мышечного тонуса в постинсультном периоде: вопросы терапии. Consilium Medicum, 2004, 6: 12-18. / Vorobieva O.V. Disturbance of muscular tonus in poststroke period: issues of therapy. Consilium Medicum, 2004, 6: 12-18.

13. Хатькова С.Е. Лечение спастичности после инсульта. Неврология, нейропсихиатрия, психосоматика, 2010, 3: 76-80. / Khatkova S.E. Treatment of spasticity after stroke. Nevrologia, neuropsikhiatria, psikhosomatika, 2010, 3: 76-80

14. Pathak M, Nguyen H, Graham H, Moore A. Management of spasticity in adults: practical application of botulinumtoxin. Eur J. Neurol, 2006, 13: 42-50.

15. Gracies JM., Singer BJ, Dunne JW. The role of botulinum toxin injections in the management of muscle overactivity of the lower limb. Disabil Rehabil, 2007, 29(23): 1789-1803.

16. Kirazli Y, On A.Y, Kismali B, Aksit R. Comparison of phenol block and botulinum toxin type $A$ in the treatment of spastic foot after stroke: a randomized, double-blind trial. Am J Phys Med Rehabil, 1998, 77: 510-515.

17. Suputtitada A, Suwanwela N. The lowest effective dose of botulinum $A$ toxin in adult patients with upper limb spasticity. Disabil Rehabil, 2005, 27: 176-184.

18. Малахов В.А. Мышечная спастичность при органических заболеваниях нервной системы и ее коррекция. Международный неврологический журнал, 2010, 5(35): 34-39. / Malakhov V.A. Muscular spasticity in organic diseases of the nervous system and its correc tion. Mezhdunarodny Nevrologichesky Journal, 2010, 5 (35): 34-39.

19. Dressler D, AdibSaberi F. Botulinumtoxin: mechanismsofaction. Eur Neurol, 2005, 53: 3-9.

20. Elia AE, Filippini G, Calandrella D, Albanese A. Botulinum neurotoxins for post-stroke spasticity in adults: a systematic review. MovDisord, 2009, 24(6): 801-812.

21. Gallichio JE Pharmacologic management of spasticity following stroke. Phys Ther, 2004, 84(10): 973-981.

22. Guidelines for the use of botulinum toxin (BTX) in the management of spasticity in adults. Clinical Effectiveness \& Evaluation, Unit. Royal College of Physicians, 2002, 17.

23. Johnson C, Burridge J, Strike P, Wood D, Swain I. The effect of combined use of botulinum toxin type $A$ and functional electric stimulation in the treatment of spastic drop foot after stroke: a preliminary investigation. Arch Phys Med Rehabil, 2004, 85: 902-909.

24. O'Brien C. Treatment of spasticity with botulinum toxin. Clin J Pain, 2002, 18: 182-190.

25. Cheung J, Rancourt A, Di Poce S, Levine A, Hoang J, Ismail F. Patient-Identified Factors That Influence Spasticity in People with Stroke and Multiple Sclerosis Receiving Botulinum Toxin Injection Treatments. Physiother Can, 2015, 67(2): 157-166

26. Кадыков А.С., Черникова Л.А., Шахпаронова Н.В. Реабилитация неврологических больных М: МЕДпресс-информ, 2008: 560. / Kadykov A.S., Chernikova L.A., Shakhparanova N.V. Rehabilitaiton of neurologic patients. $\mathrm{M}$ : MEDpress-inform, 2008:560

27. Francis H, Wade D, Turner-Stokes L., Kingswell $\mathrm{R}$, Dott $\mathrm{C}$, Coxon E. Does reducing spasticity translate into functional benefit? An exploratory meta-analysis. J Neurol Neurosurg Psychiatry, 2004, 75: 1547-1551.

28. Turkel C, Bowen B, Liu J, Brin M. Pooled analysis of the safety of botulinum toxin type $A$ in the treatment of post-stroke spasticity. Arch Phys Med Rehabil, 2006, 87: 786-792.

29. Bakheit A, Fedorova N, Skoromets A Timerbaeva S, Bhakta B, Coxon L. The benefi cial antispasticity effect of botulinum toxin type $A$ is maintained after repeated treatment cycles. J Neurol Neurosurg Psychiatry, 2004, 75: 1558-1561.

30. Brashear A, Gordon M, Elovic E, Kassicieh V Marciniak C, Do M., Botox Post-Stroke Spasticity Study Group. Intramuscular injection of botulinum toxin for the treatment of wrist and finger spasticity after a stroke. N Engl J Med, 2002, 347: 395-400.

31. Opheim A, Danielsson A, Alt Murphy M, Persson $H$, Sunnerhagen K. Early prediction of longterm upper limb spasticity after stroke: Part of the SALGOT study. Neurology, 2015, 85(10): 873-880.

32. Burbaud P, Wiart L, Dubos J, Gaujard E, Debelleix X, Joseph P. A randomized, double blind, placebo controlled trial of botulinum toxin in the treatment of spastic foot in hemi- paretic patients. J Neurol Neurosurg Psychiatry, 1996, 61: 265-269.

33. Childers M, Brashear A, Jozefczyk P, Reding M, Alexander D, Good D. Dose-dependent response to intramuscular botulinum toxin type A for upper-limb spasticity in patients after a stroke. Arch Phys Med Rehabil, 2004, 85: 1063-1069.

34. Gordon M, Brashear A, Elovic E, Kassicieh D, Marciniak C. Botox Post-Stroke Spasticity Study Group. Repeated dosing of botulinum toxin type A for upper limb spasticity following stroke. Neurology, 2004, 63: 1971-1973.

35. Hesse S, Reiter F, Konrad M, Jahnke M. Botulinum toxin type $\mathrm{A}$ and short-term electrical stimulation in the treatment of upper limb flexor spasticity after stroke: a randomized, double-blind, placebo-controlled trial. Clin Rehabil, 1998, 12: 381-388.

36. Pandyan A, Vuadens P, van Wijck F, Stark S, Johnson G, Barnes M. Are we underestimating the clinical efficacy of botulinum toxin (type A)? Ouantifying changes in spasticity, strength and upper limb function after injections of Botox to the elbow flexors in a unilateral stroke population. Clin Rehabil, 2002, 16: 654-660.

37. Rousseaux M, Kozlowski O, Froger J. Efficacy of botulinum toxin $\mathrm{A}$ in upper limb function of hemiplegic patients. J Neurol, 2002, 249: 76-84.

38. Ryuji K, Yuka O, Kazuaki S, Toshio M, Yasuyuki U. Botulinum toxin type A in post-stroke lower limb spasticity: a multicenter, double-blind, placebo-controlled trial. J Neurol, 2008, 257: 1330-1337.

39. Bayram S, Sivrioglu K, Karli N, Ozcan O. Lowdose botulinum toxin with short-term electrical stimulation in post-stroke spastic drop foot: a preliminary study. Am J Phys Med Rehabil, 2006, 85: 75-81.

40. Dengler R, Neyer U, Wohlfarth K, Bettig U., Janzik $\mathrm{H}$. Local botulinum toxin in the treatment of spastic drop foot. J Neurol, 1992, 239: 375-378.

41. Ward A. A literature review of the pathophysiology and onset of post-stroke spasticity. Eur J Neurol, 2012, 19: 21-27.

42. Hesse S, Jahnke M, Luecke D, Mauritz K. Shortterm electrical stimulation enhances the effectiveness of Botulinum toxin in the treatment of lower limb spasticity in hemiparetic patients. Neurosci Lett, 1995, 201: 37-40.

43. Pittock S, Moore A, Hardiman O. A double-blindrandomized placebo-controlled evaluation of three doses of botulinum toxin type A (Dysport). J Neurol Neurosurg Psychiatry, 1999, 58: 1345-1349.

44. Thibaut A, Chatelle C, Ziegler E, Bruno MA, Laureys S, Gosseries O. Spasticity after stroke: physiology, assessment and treatment. Brain Inj, 2013, 27: 1093-1105.

45. Opheim A, Danielsson A, Alt Murphy M, Persson HC, Sunnerhagen K. Upper limb spasticity during the first year after stroke: a longitudinal study at the University of Gothenburg (SALGOT). Am J Phys Med Rehabil, 2014, 93: 884-896.

46. Костенко Е.В., Петрова Л.В., Ганжула П.Н., Хозова А.А., Бойко А.Н. Опыт применения ксеомина в коррекции спастичности руки в ранний восстановительный период инсульта. Журнал неврологии и психиатрии им. С.C. Корсакова, 2012, 112, 2: 29-3. / Kostenko E.V., Petrova L.V., Ganzhula P.N., Khozova A.A., Boyko A.N. Experience of xeomin use for correction of hand spasticity at an early rehabilitation period after stroke. Zhurnal Nevrologii I Psikhiatrii im. S.S. Korsakova, 2012, 112, 2:29-3. 